\title{
Discriminating Healthy Controls and Two Clinical Subgroups of Nonspecific Chronic Low Back Pain Patients Using Trunk Muscle Activation and Lumbosacral Kinematics of Postures and Movements
}

\author{
A Statistical Classification Model
}

\author{
Wim Dankaerts, $\mathrm{PhD},{ }^{*} \dagger$ Peter 0 'Sullivan, PhD, $\neq$ Angus Burnett, PhD, $\mathbb{S}$ Leon Straker, PhD, $\ddagger$ \\ Paul Davey, BSc, $\ddagger$ and Ritu Gupta, PhD $\ddagger$
}

\begin{abstract}
Study Design. Statistical Classification Model for nonspecific chronic low back pain (NS-CLBP) patients and controls based on parameters of motor control.

Objective. Develop a Statistical Classification Model to discriminate between 2 subgroups of NS-CLBP (Flexion Pattern [FP] and Active Extension Pattern [AEP]) and a control group using biomechanical variables quantifying parameters of motor control.
\end{abstract}

Summary of Background Data. It has been well documented that many CLBP patients have motor control impairments of their lumbar spine. O'Sullivan proposed a mechanism-based classification system for NS-CLBP with motor control impairments based on a comprehensive subjective and physical examination to establish the relationship between pain provocation and spinal motor control. For the FP and AEP s, 2 groups defined by $\mathrm{O}$ 'Sullivan and under investigation is this study, the motor control impairment is considered to be the mechanism maintaining their CLBP. No previous studies have used a Statistical Model with measurements of motor control impairment to subclassify NS-CLBP patients.

Methods. Thirty-three NS-CLBP patients (20 FP and 13 $\mathrm{AEP}$ ) and 34 asymptomatic subjects had synchronized lumbosacral kinematics and trunk muscle activation recorded during commonly reported aggravating postures and movements. Biomechanical variables were quantified and a Statistical Classification Model was developed.

Results. The Statistical Model used 5 kinematic and 2 electromyography variables. The model correctly classified $96.4 \%$ of cases.

Conclusion. Selected biomechanical variables were predictors for subgroup membership and were able to

From the "Musculoskeletal Unit, Department of Rehabilitation Sciences, Faculty of Kinesiology and Rehabilitation Sciences K.U. Leuven, Leuven, Belgium; †Department of Health Care, University College Limburg, AUHL-PHL, REVAL-Rehabilitation and Health Care Research Center, Hasselt, Belgium; $\ddagger$ School of Physiotherapy, Curtin University of Technology, Perth, Western Australia; and \$School of Exercise, Biomedical, and Health Sciences, Edith Cowan University, Joondalup, Western Australia.

Acknowledgment date: January 20, 2009. Acceptance date: January 20, 2009.

The manuscript submitted does not contain information about medical device $(\mathrm{s}) / \mathrm{drug}(\mathrm{s})$.

Federal and Institutional funds were received in support of this work. No benefits in any form have been or will be received from a commercial party related directly or indirectly to the subject of this manuscript. Address correspondence and reprint requests to Wim Dankaerts, PhD, Musculoskeletal Unit, Department of Rehabilitation Sciences, Faculty of Kinesiology and Rehabilitation Sciences, K.U. Leuven, Tervuursevest 101,B-3001 Leuven, Belgium; E-mail: wim.dankaerts@ faber.kuleuven.be discriminate the 3 subgroups. This study adds further support toward the validation of the proposed classification system.

Key words: low back pain, subclassification, electromyography, motion analysis, posture, motor control impairment. Spine 2009;34:1610-1618

Low back pain (LBP) is one of the most common and costly musculoskeletal pain syndromes of modern society with up to $80 \%$ of people having LBP at some time in their life. ${ }^{1,2}$ LBP often develops into a chronic fluctuating problem with intermittent flares. ${ }^{3,4}$ In up to $85 \%$ of cases there is no radiologic abnormality evident, so many patients are classified as having nonspecific chronic LBP (NS-CLBP). ${ }^{5,6}$ The lack of success in defining subgroups of patients has been offered as an explanation for the inability to identify effective treatments for NS-CLBP., ${ }^{7,8}$ Thus, there have been strong recommendations to establish methods of classification that distinguish subgroups of LBP from each other. ${ }^{7-9}$

It is now well accepted that LBP is a bio-psycho-social problem. ${ }^{6,10}$ Recent critical appraisals and systematic reviews highlight that the majority of studies that relate to the classification of LBP have focused on only a single dimension of the LBP problem, rather than consideration being given to its multiple dimensions. ${ }^{11-14} \mathrm{~A}$ multidimensional classification system has been proposed by O'Sullivan ${ }^{15,16}$ for a subgroup of NS-CLBP where altered motor control is suggested to represent the underlying driving mechanism for the pain. It is hypothesized that these subjects present with maladaptive motor behavior that exposes their spine to ongoing strain leading to pain. This classification system describes 5 distinct directional patterns of motor control impairment: Flexion Pattern (FP), Active Extension Pattern (AEP), Passive Extension Pattern, Lateral Shifting Pattern, and MultiDirectional Pattern. It is suggested that each of these patterns represent a subgroup of the larger NS-CLBP population and a different intervention is proposed for each pattern. ${ }^{15,16}$

The subgroups under investigation in this study are the FP and AEP because previous research ${ }^{17}$ has established these are the 2 most common. Table 1 lists the clinical features of both patterns. For a more detailed description of all patterns see O'Sullivan. ${ }^{15,16}$ 
Table 1. Inclusion Criteria and Clinical Features of LBP Subjects

Inclusion criteria-NS-CLBP with motor control impairment of FP or AEP $>3$-mo nonspecific LBP

Revised Oswestry score $>15 \%$

Pain localized to the lower lumbar spine ( $L 4 / L 5$ or $L 5 / S 1)$ region

Absence of "red flags" (specific causes of LBP such as cauda equina syndrome or inflammatory disease)

Absence of dominant "yellow flags" (identification of beliefs, emotions, and behaviors that interact with the pain problem)

Clear mechanical basis of the disorder (pain related to postures and movements)

Associated impairments in the control of the motion segment(s) in the provocative movement direction(s)

Absence of impaired movement of the symptomatic segment in the painful direction of movement or loading (based on clinical joint motion palpation examination)

Clinical diagnosis of a FP or AEP disorder, both clinicians (independently) agreed on the diagnosis

Key clinical features-FP

Aggravation of symptoms with movements and postures involving flexion of the lower lumbar spine

Loss of segmental lordosis at symptomatic level with posture and movement, difficulty assuming, and/or maintaining neutral lordotic postures with a tendency to flex lower lumbar spine

Decreased muscle tone in sLM assessed by manual palpation

Pain relief with spinal extension

Key clinical features-AEP

Aggravation of symptoms with movements and postures involving extension of the lower lumbar spine

Excessive segmental lordosis at symptomatic level with posture and movements

Difficulty assuming and/or maintaining neutral lordotic postures with a tendency to position themselves into hyperextension

Increased muscle tone in sLM assessed by manual palpation

Pain relief with spinal flexion

From this research it is also clear that trained clinicians (physiotherapists and medical physicians) can reliably identify these subgroups. ${ }^{17}$ There is also growing support for the validity of these patient subgroups with recent studies revealing altered spinal repositioning sense, adopting potentially provocative end range spinal postures during sitting, altered spinal kinematics, and abnormal muscle activation patterns among subgroups consistent with the proposed classification system. ${ }^{18-22}$

Careful analysis of spinal postures and movements, reported to be provocative by the patient, are an important component of the proposed clinical classification system as it evaluates how these factors influence the specific presentation of LBP (in terms of provocation or relief of pain). ${ }^{15}$ A process of "diagnostics" is used to establish the relationship between pain provocation and spinal motor control to enhance the accuracy of the classification system. ${ }^{23}$

However, these clinical assessments have not been formally investigated. The logical next step in establishing the validity of this classification system is to determine whether spinal postures and movements do in fact discriminate between clinically determined subgroups with NSCLBP and healthy controls.

Synchronized recording of electromyography (EMG) of selected trunk muscles and spinal kinematics have been used frequently in the literature to describe the motor control of LBP patients compared with pain-free controls. ${ }^{24-28}$ However, to date no studies have used such an approach to subclassify the NS-CLBP population. Therefore, the aim of this study was to use these biomechanical approaches to quantify parameters of motor control. More specifically, the objective of the study was to develop a Statistical Classification Model and test its ability to discriminate 2 subgroups of NS-CLBP (FP and AEP) and a subgroup of pain-free controls using trunk muscle activation and lumbosacral kinematics during postures and movements, which were reported as pain aggravating in these clinical subgroups.

\section{- Materials and Methods}

Motor control, as assessed by trunk muscle activation (EMG) and lumbosacral kinematics, was recorded from subjects with no-LBP and 2 clinically diagnosed subgroups of subjects with NS-CLBP (FP and AEP) during common postures and movements. Data were used to develop a Statistical Classification Model to compare the laboratory testing with the clinical diagnosis. Figure 1 outlines the experimental protocol.

\section{Participants}

Sixty-seven subjects participated in this study of which 34 were healthy controls (no-LBP) and 33 had NS-CLBP with a classification of motor control impairment. Control subjects were a convenience sample and consisted of University students, relatives of students, and personnel affiliated with the University. They were excluded from the study if they had a history of LBP or leg pain over the previous 2 years and/or had received previous postural education. NS-CLBP patients were recruited
Figure 1. Flow-chart of study protocol developing a model based on laboratory derived measures for motor control to discriminate and subclassify subjects and testing its accuracy versus the clinical examination ("gold standard").

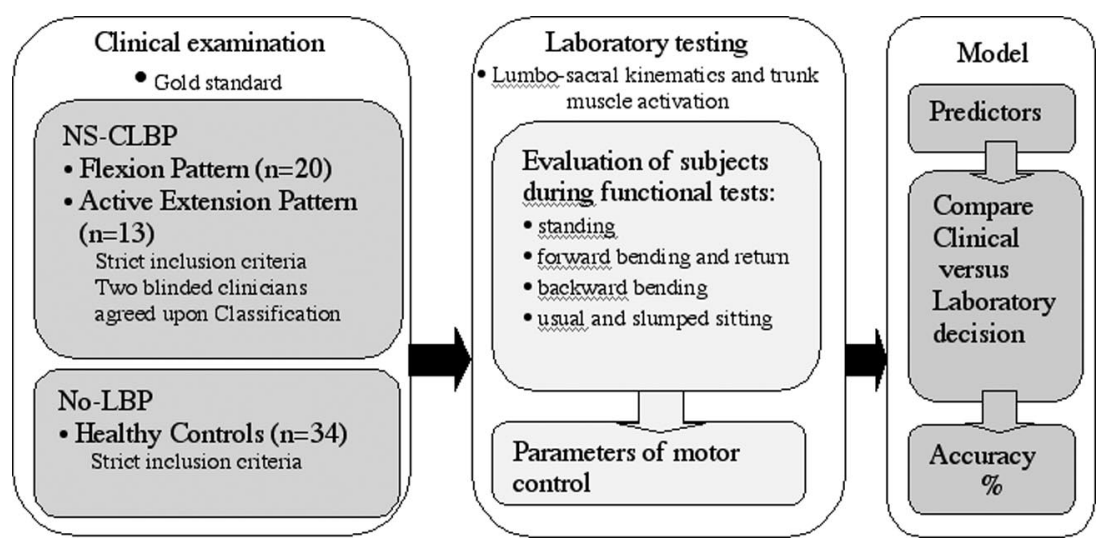


Table 2. Characteristics of Subjects per Group; Count (\%) or Mean (SD)

\begin{tabular}{|c|c|c|c|c|}
\hline & No LBP Controls $(n=34)$ & Flexion Pattern $(n=20)$ & $\operatorname{AEP}(n=13)$ & Group Differences Sign \\
\hline Gender & & & & ${ }^{*} \chi^{2}=6.4 ; P=0.04$ \\
\hline Males & $18(53 \%)$ & $16(80 \%)$ & $5(38 \%)$ & - \\
\hline Females & $16(47 \%)$ & $4(20 \%)$ & $8(61.5 \%)$ & - \\
\hline Age (yr) & $32.0(12.2)$ & $35.7(11.2)$ & $39.9(11.3)$ & $\mathrm{F}_{2,67}=2.3 ; P=0.11$ \\
\hline Weight (kg) & $68.4(11.6)$ & $80.1(10.6)$ & $72.8(15.7)$ & ${ }^{*} \mathrm{~F}_{2,67}=5.7 ; P=0.005$ \\
\hline Height $(m)$ & $1.71(0.10)$ & $1.80(0.10)$ & $1.70(0.10)$ & $\mathrm{F}_{2,67}=1.5 ; P=0.23$ \\
\hline BMI $\left(\mathrm{kg} / \mathrm{m}^{2}\right)$ & $23.3(2.9)$ & $24.6(2.5)$ & $24.2(2.8)$ & $\mathrm{F}_{267}=1.8 ; P=0.17$ \\
\hline VAS (24 h/10) & - & $4.2(1.9)$ & $5.7(2.1)$ & $t=1.9 ; P=0.06$ \\
\hline R-0swestry (\%) & - & $36.6(11.0)$ & $41.2(14.2)$ & $t=1.01 ; P=0.20$ \\
\hline TSK & - & 40.2 (8.2) & 41.3 (8.8) & $t=0.36 ; P=0.72$ \\
\hline
\end{tabular}

${ }^{*} P<0.05$.

BMI indicates body mass index; VAS, Visual Analogue Scale; R-Oswestry, revised Oswestry ${ }^{29}$; TSK, Tampa Scale of Kinesiophobia. ${ }^{30}$

from a private orthopaedic clinic. Two clinicians (musculoskeletal physiotherapists) identified and subclassified the subjects with NS-CLBP in a blinded manner. Patients were included in the study when there was mutual agreement between clinicians on a classification of FP or AEP (referred to as the gold standard). For logistical reasons, even though 3 other patterns of motor control impairment are proposed, only patients with a classification of FP and AEP were selected for this study. The strict inclusion criteria for these subgroups and the characteristics of subjects in each subgroup are described in Tables 1 and 2 , respectively. NS-CLBP patients underwent the laboratory testing within a week of the clinical examination. Before testing, all subjects read and signed a consent form, which outlined the testing protocol. The Human Research Ethics Committee of Curtin University, Western Australia approved the study.

\section{Experimental Protocol-Series of Functional Postures and Movements}

Laboratory testing consisted of synchronized recordings of the activation of 3 superficial trunk muscles and lumbosacral kinematics obtained from a series of postures and movements known to aggravate LBP. These postures and movements were; standing, forward bending and return, backward bending and usual, and slumped sitting. The instructions used for testing are listed in Table 3. For all tasks the average of 3 trials (each 5 seconds in duration) was used for further analysis. Range of movement data were time normalized into quartiles of movement $(0 \%-25 \%, 25 \%-50 \%$ etc.). To control the timing of all movements a metronome was used.

\section{Data Collection and Management}

Lumbosacral Kinematics. Lumbosacral kinematic data were recorded using the 3-Space Fastrak (Polhemus Navigation Science Division, Kaiser Aerospace, VT). The Fastrak system is a noninvasive electromagnetic device, which measures the position and orientation of points in space. This apparatus has been shown to be both reliable and valid for measurement of transducer movement with an accuracy of $0.2^{\circ}{ }^{31}$ Sensors were placed on the skin over the spinous processes of T12, L3, and S2 using double-sided tape (Norton Pty Ltd, NSW, Australia) and Fixomull sports tape (Beiersdorf AG, Hamburg, Germany) with the participant in slight spinal flexion to minimize displacement caused by skin movement.

Data were collected at $25 \mathrm{~Hz}$ using a customized program written in LabVIEW V6.1 (National Instruments, Richardson, TX). Three lumbosacral angles were calculated in the sagittal plane as follows.
- Sacral angle: inclination of the sensor at $\mathrm{S} 2$ relative to the vertical. ${ }^{18,32}$ A larger positive angle indicates more posterior tilt of the sacrum.

- Lower lumbar angle (LLx): the angle between 2 intersecting lines, one indicating the inclination of the sensor at L3 and the other the inclination of the sensor at S2. A negative LLx indicates lumbar lordosis. ${ }^{18}$

- Lumbar angle (Lx): the angle between 2 intersecting lines, one indicating the inclination of the sensor at T12 and the other the inclination of the sensor at S2. A negative Lx indicates lumbar lordosis. ${ }^{18}$

Before processing the raw data, a customized quality control program in conjunction with visual inspection was used to detect and eliminate movement artifact. The above-mentioned angles were calculated during the series of functional tests.

Trunk Muscle Activation. Trunk muscle activation (EMG) data were sampled using an 8 channels Octopus Cable Telemetric system (Bortec Electronics Inc., Calgary, Canada) at 1000 $\mathrm{Hz}$. The bandwidth was 10 to $500 \mathrm{~Hz}$, and the common mode rejection ratio was $>115 \mathrm{~dB}$ at $60 \mathrm{~Hz}$. All raw myoelectric signals were preamplified and amplified with an overall gain of

\section{Table 3. Description of Postures and Movements (With Related Instructions) Used in Experimental Protocol}

Task Instructions Given to Subjects

Standing

Forward bending and return

"Stand still with feet shoulder-width apart, knees straight and arms hanging freely, look forward."

"With feet shoulder-width apart, knees straight and arms hanging freely bend forward in 5 sec as far as possible, like touching your toes, pause for $5 \mathrm{sec}$ at the end, and then rise in 5 sec to upright posture, keep knees straight all the time."

Backward "With feet shoulder-width apart, knees straight bending and arms hanging freely, bend backwards in 5 sec as far as possible, pause for 5 sec at the end."

Usual sitting* "Sit how you would usually sit on a stool like this."

Slumped sitting* "Sit and slouch your back, try to fully relax."

* Participants sat on a stool (with no back support) with a flat, horizontal surface. The height of the stool was adjusted to ensure that the participants' upper legs were horizontal and the lower legs vertical. The feet were positioned shoulder width apart with arms hanging relaxed next to the thighs. Participants viewed a visual target set $1.5 \mathrm{~m}$ in front, at eye level, to standardize the head posture. 
2000. Data were collected on a computer running LabVIEW V6.1 (National Instruments). Pairs of self-adhesive disposable $\mathrm{Ag} / \mathrm{AgCl}$ (Red Dot, $3 \mathrm{M}$ Health Care Products, London, Canada) disc surface electrodes with an electrical contact surface of $1 \mathrm{~cm}^{2}$ were placed parallel to the muscle fibers with a centerto-center spacing of $2.5 \mathrm{~cm}$. Snap leads were used to connect the surface electrodes to the preamplifiers. Skin preparation for sEMG was according to Hermens et al. ${ }^{33,34}$

Pairs of surface electrodes were bilaterally positioned over 3 trunk muscles as follows:

- Transverse fibers of internal oblique: $1 \mathrm{~cm}$ medial to the anterior superior iliac spine and beneath a line joining both anterior superior iliac spines. ${ }^{35}$

- Superficial fibers of lumbar multifidus (sLM): at L5 level and aligned parallel to the line between the posterior superior iliac spine and the L1-L2 interspinous space. ${ }^{36}$

- Iliocostalis lumborum pars thoracis: above and below the level of L1 spinous process midway between the midline and the lateral aspect of the body. ${ }^{37}$

- A common earth electrode was placed over the left iliac crest.

Before processing the raw sEMG data, a customized program in conjunction with visual inspection was used to detect and eliminate possible contamination by heartbeat and other artifacts. Raw data were then demeaned, full-wave rectified, and band pass filtered ( 4 and $400 \mathrm{~Hz}$ ) using a fourth-order zero lag Butterworth filter ${ }^{38}$ and a linear envelope was calculated for each channel.

Surface EMG measurements for abdominal and back muscles were amplitude normalized to 2 standardized activities designed to elicit a stable submaximal voluntary isometric contraction (sub-MVIC). These normalization procedures have been described in detail elsewhere ${ }^{39}$ and have been shown to be reliable both within-day and between-days.

Parameters of Motor Control Used to Develop the Statistical Classification Model. Several parameters describing aspects of motor control of the lumbar spine were derived from laboratory testing. Table 4 provides an overview of all derived trunk muscle activation and lumbosacral kinematic variables. These parameters of motor control were based on those considered to be most clinically important for the clinical decision-making regarding the classification system ${ }^{15}$ and were used to develop a Statistical Classification Model (see Statistical analysis). For all sEMG variables statistical analyses were performed for left and right sides. Preliminary analysis revealed that there was no difference between sides, hence data from one randomly selected side (left) were subsequently used.

Reliability of the Laboratory Measurements. The intertrial reliability ${ }^{40}$ was assessed on all subjects and was excellent. For the lumbosacral data, intraclass correlation coefficients ${ }_{(3,1)}$ ranged between 0.85 and 0.99 and the standard error of measurement ranged from $0.7^{\circ}$ to $5.1^{\circ}$. For sEMG data, intraclass correlation coefficients ranged between 0.87 and 0.99 and the standard error of measurement ranged from 0.05 to 0.18 ( $\%$ of sub-MVIC).

\section{Statistical Analysis}

Gender, age, weight, and body mass index were used as independent variables in all analyses. The ability of selected parameters of motor control to discriminate between subgroups (noLBP, FP, or AEP) compared with the clinical classification (considered as the gold standard) was analyzed. Step-wise lin-
Table 4. Overview of Parameters of Motor Control*

\begin{tabular}{|c|c|c|c|c|c|c|}
\hline & \multicolumn{3}{|c|}{ EMG Variables } & \multicolumn{3}{|c|}{$\begin{array}{l}\text { Kinematic } \\
\text { Variables }\end{array}$} \\
\hline & Trlo & sLM & ICLT & SA & LLx & $\mathrm{Lx}$ \\
\hline Standing & $\nu$ & $\nu$ & $\nu$ & $\nu$ & $\nu$ & $\nu$ \\
\hline \multicolumn{7}{|l|}{ Forward bending } \\
\hline 01 & $\nu$ & $\nu$ & $\nu$ & $\nu$ & $\nu$ & $\nu$ \\
\hline 02 & $\nu$ & $\nu$ & $\nu$ & $\nu^{\dagger}$ & $\nu$ & $\nu$ \\
\hline 03 & $\nu$ & $\nu$ & $\nu$ & $\nu$ & $\nu$ & $\nu$ \\
\hline 04 & $\nu$ & $\nu$ & $\nu$ & $\nu$ & $\nu^{*}$ & $\nu$ \\
\hline Fully bent & $\nu$ & $\nu^{*}$ & $\nu$ & $\nu$ & $\nu$ & $\nu$ \\
\hline FRR & $\nu$ & $\nu$ & $\nu$ & & & \\
\hline \multicolumn{7}{|c|}{ Return from forward bending } \\
\hline 01 & $\nu$ & $\nu$ & $\nu$ & $\nu$ & $\nu$ & $\nu$ \\
\hline 02 & $\nu$ & $\nu$ & $\nu$ & $\nu^{*}$ & $\nu$ & $\nu$ \\
\hline 03 & $\nu$ & $\nu$ & $\nu$ & $\nu$ & $\nu$ & $\nu$ \\
\hline 04 & $\nu$ & $\nu$ & $\nu$ & $\nu$ & $\nu$ & $\nu$ \\
\hline \multicolumn{7}{|l|}{ Backward bending } \\
\hline 01 & $\nu$ & $\nu$ & $\nu$ & $\nu$ & $\nu$ & $\nu$ \\
\hline 02 & $\nu$ & $\nu$ & $\nu$ & $\nu$ & $\nu$ & $\nu$ \\
\hline 03 & $\nu$ & $\nu$ & $\nu$ & $\nu$ & $\nu$ & $\nu$ \\
\hline 04 & $\nu$ & $\nu$ & $\nu$ & $\nu$ & $\nu$ & $\nu$ \\
\hline Fully bent & $\nu$ & $\nu$ & $\nu$ & $\nu$ & $\nu$ & $\nu$ \\
\hline ERR & $\nu$ & $\nu$ & $\nu$ & & & \\
\hline \multicolumn{7}{|l|}{ Sitting } \\
\hline Usual sitting & $\nu$ & $\nu$ & $\nu$ & $\nu^{*}$ & $\nu$ & $\nu^{*}$ \\
\hline Slumped sitting & $\nu$ & $\nu$ & $\nu$ & $\nu$ & $\nu$ & $\nu$ \\
\hline Usual slumped & $\nu$ & $\nu$ & $\nu$ & $\nu$ & $\nu$ & $\nu$ \\
\hline FRR & $\nu$ & $\nu^{*}$ & $\nu$ & - & - & - \\
\hline
\end{tabular}

* Trunk muscle activation (EMG) and lumbosacral kinematic variables used in developing the statistical classification model.

† Used in final multivariate model (= predictors for subgroup membership). $\mathrm{Q}$ indicates time normalized quartile; TrlO, transverse fibers of internal oblique; ICLT, iliocostalis lumborum pars thoracis; SA, sacral angle; FRR, flexion relaxation ratio; FRR sitting, average sEMG in usual sitting divided by average activity in slumped sitting; FRR forward bending, average sEMG in standing divided by average activity in full forward bending; ERR, Extension Relaxation Ratio; ERR backwards bending, average sEMG in standing divided by average activity in full backward bending.

ear discriminant analysis (LDA) was used to develop a Statistical Classification Model on all valid cases $(n=53) .{ }^{41}$ A valid case was considered where a subject had no missing data from the laboratory testing. LDA is a mathematical procedure to determine which variables (referred to as "predictors") discriminate between subgroups. ${ }^{42}$ LDA computes classification functions that determine which subgroup each case most likely belongs. Two functions were generated by this analysis as the number of functions generated is equal to $k-1$, where $k$ is the number of subgroups. ${ }^{42}$ Each function allowed the computation of classification scores for each case with respect to each subgroup. For each set of classification functions generated the correlation between search variables were computed. None of the variables included in the analysis presented high correlations.

Two methods were used to test the model's validity. First, a cross-validation procedure (leave-one-out method) was used to validate the Model based on all valid cases. ${ }^{42}$ Second, a holdout validation was used to further test the robustness of the Model. For the hold-out validation, 5 different random samples (sample size $\pm 66 \%$ ) were selected from all cases. The sample selected (learning sample) and the cases not included in this sample constituted a hold-out sample. The Statistical Classification Model was then developed for each learning sample and its accuracy in classifying the hold-out sample was determined and expressed as a percentage of correctly classified cases.

To enable interpretation of the model, standardized canonical discriminant function coefficients and standardized canon- 
ical discriminant scores were calculated, and univariate analysis was performed on each of the predictors independently (descriptive statistics; ANOVA and post hoc Bonferroni; $P<$ 0.05). All analyses were performed using SPSS V12.0.

\section{- Results}

\section{Multivariate Discrimination of Three Subgroups Using Parameters of Motor Control}

Statistical Classification Model. Of the 117 parameters of motor control outlined in Table 4 the LDA produced 7 predictors that were entered into the final Statistical Classification Model (variables A-G in Table 5). The model developed was as follows: indicates that the 7 variables are strong predictors for subgroup membership.

Model Accuracy and Validation. Table 6 reports on the accuracy of the statistical classification model to subclassify the subjects in this study as no-LBP, FP, or AEP. These data indicate that a combination of 7 parameters of motor control entered into the model resulted in correct classification in $96.4 \%$ of cases based on the clinical decision-making. It is notable that these results were relatively consistent among the 2 validation methods used (cross-validation: $94.6 \%$; hold-out validation: $74.5 \%$ ) (Table 6). This indicates that the model is robust and capable of accurately discriminating between the sub-

$$
\left\{\begin{array}{l}
\hat{d}_{\mathrm{NO}-\mathrm{LBP}}=0.77 \times A-0.82 \times B-0.4 \times C-5.13 \times D+0.20 \times E-5.13 \times F+3.75 \times G-33.07 \\
\hat{d}_{\mathrm{AEP}}=0.35 \times A-1.14 \times B-0.05 \times C-6.35 \times D+0.579 \times E-6.35 \times F+2.11 \times G-32.01 \\
\hat{d}_{\mathrm{FP}}=0.27 \times A-0.65 \times B-0.28 \times C-1.4 \times D+0.53 \times E-1.4 \times F+1.72 \times G-17.47
\end{array}\right.
$$

where $\hat{d}_{i} ; i=N o-L B P, A E P, F P$ were the discriminant scores and $A-G$ were the measurements on the 7 variables. A new case was allocated to the subgroup based on their largest discriminant score.

Standardized Canonical Discriminant Function Coefficients and Scores. The standardized canonical discriminant function coefficients for the model are presented in Table 5 and show the loadings for each variable on the 2 functions. It can be seen that function 1 was dominated by the sEMG activity of the sLM at the end of forward bending and the lower lumbar angle during the last quarter of forward bending. Function 2 was dominated by the posture in sitting (lumbar curvature). Function 1 accounted for $72.4 \%$ of the discriminant variance (Wilks lambda $=$ $0.05 ; P<0.0001$ ) whereas function 2 accounted for an additional $27.6 \%$ of the variance (Wilks lambda $=0.33$; $P<0.0001)$.

Plots of standardized canonical discriminant scores for each subject are represented in Figure 2. This figure clearly shows a distinct clustering of the 3 subgroups and

Table 5. Standardized Canonical Discriminant Function Coefficients

\begin{tabular}{lrr}
\hline & Function 1 & Function 2 \\
\hline Canonical correlation & 0.92 & 0.82 \\
Eigenvalue & 5.34 & 2.03 \\
Predictive variables & & \\
A Forward bending LLx 04 & -0.97 & -0.69 \\
B Forward bending SA 02 & -0.20 & 0.81 \\
C Return Forward bending SA 02 & 0.74 & -0.55 \\
D Usual sitting Lx & -0.01 & 1.00 \\
E Usual sitting SA & 0.69 & 0.23 \\
F Forward bending sLM fully bend & 1.06 & -0.11 \\
G FRR LM in sitting & -0.58 & -0.44 \\
\hline
\end{tabular}

groups as determined by the 2 clinicians.

The classification accuracy was compared for each block of variables (lumbosacral kinematics and sEMG). Separate models based only on lumbosacral kinematics or trunk muscle activation variables showed a decrease in accuracy of approximately 10\% (Table 6).

Differences Between Subgroups Based on Univariate Analysis. Results indicated that the subgroups differed significantly on all predictors (Table 7). In summary, these results showed greater sLM activity for the AEP subgroup at the end of forward bending compared with the FP subgroup, with the FP subgroup typified by increased activation of sLM compared with the no-LBP group.

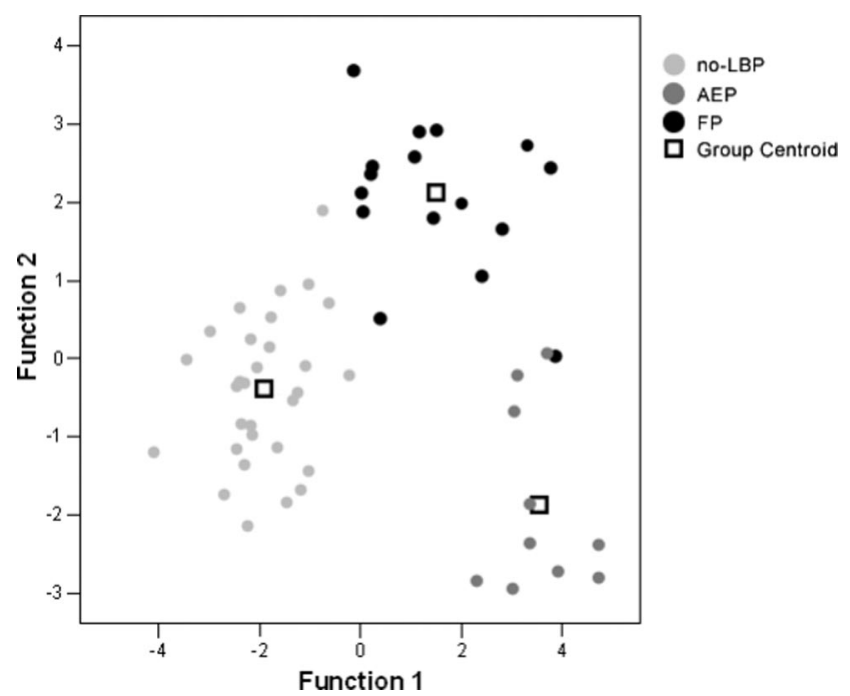

Figure 2. Figure 2 shows plot of standardized canonical discriminant scores for all subjects (FP; AEP). Because there were 2 subgroups in this study, 2 functions were generated. See Table 5 for loading values of variables on the functions. 
Table 6. Model, Cross-Validation, and Hold-Out Group Validation Accuracy (\%)

\begin{tabular}{lcc}
\hline & $\begin{array}{c}\% \\
\text { Accuracy (Range) }\end{array}$ & $\begin{array}{c}\text { Valid Cases } \\
\text { (n) (no LBP, } \\
\text { AEP, FP Groups) }\end{array}$ \\
\hline $\begin{array}{c}\text { Kinematics + sEMG } \\
\text { Statistical classification model } \\
\text { Model validation } \\
\quad \text { Cross-validation } \\
\quad \text { Hold-out validation }\end{array}$ & 96.4 & $29,10,14$ \\
$\begin{array}{c}\text { Kinematics only } \\
\text { Statistical classification model } \\
\text { Model validation } \\
\quad \text { Cross-validation }\end{array}$ & $74.5(53.9-93.8)$ & $\begin{array}{c}29,10,14 \\
\quad \text { Hold-out validation }\end{array}$ \\
$\begin{array}{c}\text { sEMG all cases } \\
\text { Statistical classification model } \\
\quad \text { Model validation } \\
\quad \text { Cross-validation } \\
\text { Hold-out validation }\end{array}$ & 87.3 & $34,13,16$ \\
\hline
\end{tabular}

This muscle activation pattern was associated with the AEP subgroup maintaining a lordotic posture (no curve reversal) at the end of forward bending and the no-LBP and FP subgroups being equally flexed (kyphotic) lumbar spine. The FP subgroup presented with more posterior rotation of the pelvis during forward bending than the AEP and no-LBP subgroups. Further, the AEP subgroup sat with a more extended lumbar spine and the FP subgroup with a more flexed lumbar spine, compared with the no-LBP subgroup. Finally, the results showed an absence of Flexion Relaxation in sitting (Flexion Relaxation in sitting $\geq 1$ ) in the 2 clinical subgroups (AEP and FP) when compared with the no-LBP subgroup. These findings are consistent with the proposed clinical classification of these subgroups.

\section{- Discussion}

This study demonstrated differences between the 3 clinically identified subgroups (no-LBP, AEP, and FP) in parameters of motor control (trunk muscle activation and lumbosacral kinematics), which concurs with the classification system proposed by O'Sullivan. ${ }^{15,16,43}$ This mul- tidimensional classification system, like some other clinical classification systems for $\operatorname{LBP}\left(e . g .,^{13,44-46}\right)$ is based on a judgmental approach. Such an approach relies primarily on the clinical insights of the developer linked with an understanding of the bio-psycho-social nature of chronic back pain disorders, which increases the potential for personal bias. ${ }^{14,47}$ Hence, it is critical to investigate the validity of the proposed classification system with quantitative measures. Using a Statistical Classification Model, this study demonstrated that clinical subgroups of NS-CLBP patients with a classification of motor control impairment as determined by 2 independent clinicians, could be discriminated from a subgroup of pain-free controls, and each other, based on selected parameters of motor control. These findings are consistent with the clinical criteria for the proposed classification system ${ }^{15}$ further strengthening the validity of this system.

The multivariate analysis showed that function 1 maximally separated the no-LBP subjects from the AEP and FP subgroups (Figure 2). Dominators (as indicated by standardized canonical discriminant function coefficients; Table 5) in function 1 were activation of the sLM at full flexion and the magnitude of the lower lumbar angle in the last quartile of forward bending. Univariate analysis demonstrated different patterns between the 3 subgroups for the activity of the sLM at full flexion (Table 7). The no-LBP subgroup presented with only $17 \%$ (sub-MVIC normalized) activity of sLM in full flexion and the 2 clinical subgroups showed significantly higher levels of activation, yet these were also distinctly different to each other $(\mathrm{AEP}=117 \%$; FP $=87 \%)$. Based on the definition of Flexion Relaxation Phenomena (FRP), a high level of muscular activity at end range of forward bending will indicate its absence. ${ }^{48,49}$

Although several other studies have shown the ability to discriminate no-LBP from LBP subjects on the basis of FRP during forward bending (e.g., Watson $e t a l^{48}$ ) the current study identified 2 distinct patterns of FRP between the FP and AEP subgroups. Combining the differences in trunk muscle activation during forward bending and the related lumbosacral kinematics strengthened the

Table 7. Univariate Analysis for Each Predictor Used in the Statistical Classification Model*

\begin{tabular}{|c|c|c|c|c|c|c|}
\hline \multirow[b]{2}{*}{ Predictor } & \multirow[b]{2}{*}{ No LBP } & \multirow[b]{2}{*}{ AEP } & \multirow[b]{2}{*}{ FP } & \multicolumn{2}{|c|}{ ANOVA } & \multirow[b]{2}{*}{ Post Hoc Bonferron } \\
\hline & & & & $F$ & $P$ & \\
\hline Forward bending LLx $04\left(^{\circ}\right)$ & $8.4(7.6)$ & $-6.6(12.8)$ & $6.1(9.7)$ & 12.2 & $<0.001 \dagger$ & $\mathrm{AEP}<\mathrm{FP}=$ no LBP \\
\hline Forward bending SA $02\left(^{\circ}\right)$ & $-34.6(7.2)$ & $-36.9(6.5)$ & $-26.8(6.6)$ & 9.5 & $<0.001 \dagger$ & $\mathrm{FP}>\mathrm{AEP}=$ no LBP \\
\hline Return forward bending SA $02\left(^{\circ}\right)$ & $-45.3(11.4)$ & $-39.3(11.5)$ & $-36.7(6.3)$ & 4.3 & $<0.018 \dagger$ & $\begin{array}{l}\text { No } L B P=A E P \\
\text { No LBP }<\text { FP } \\
\text { AEP }=F P\end{array}$ \\
\hline Usual sitting $\operatorname{Lx}\left({ }^{\circ}\right)$ & $-10.8(10.3)$ & $-26.4(13.6)$ & $1.9(6.9)$ & 29.2 & $<0.001 \dagger$ & $\mathrm{AEP}<$ no $\mathrm{LBP}<\mathrm{FP}$ \\
\hline Usual sitting $\mathrm{SA}\left({ }^{\circ}\right)$ & $-11.2^{\circ}(9.9)$ & $-17.4^{\circ}(9.6)$ & $2.2^{\circ}(7.3)$ & 20.4 & $<0.001 \dagger$ & $\mathrm{AEP}=$ no LBP $<\mathrm{FP}$ \\
\hline Forward bending fully bent sLM ( $\%$ subMVIC) & $17(13)$ & $117(89)$ & $87(67)$ & 36.3 & $<0.001 \dagger$ & $\mathrm{AEP}>\mathrm{FP}>$ no LBP \\
\hline Sitting FRR LM & $1.4(0.6)$ & $1.0(0.4)$ & $0.9(0.3)$ & 3.7 & $<0.031 \dagger$ & No $L B P>A E P=F P$ \\
\hline
\end{tabular}

${ }^{*}$ Data are reported as mean $( \pm S D)$.

$\dagger P<0.05$.

All kinematic variables in degrees; for $L x$ and LLx, a more negative value indicates more lordosis; for SA a more negative value indicates more anterior tilting of sacrum (increased lordosis); sEMG in \% of submaximal activity; FRR expressed as a ratio usual/slouched sitting. 
proposition that there exists 2 different underlying motor control patterns in NS-CLBP subjects (the AEP and FP subgroups).

High levels of activation of the sLM in the AEP subgroup were associated with an extended (lordotic) spinal posture and no curve reversal at the end of forward bending (Table 7). This demonstrates that the AEP subgroup actively posture their spine in hyperextension. Hyperextension has the potential to induce muscle fatigue and increased compressive loading to the posterior structures via forces generated by the activity of the extensor muscles. ${ }^{50}$ The AEP subgroup had reported their pain was aggravated with extension-related postures and activities while relief of pain was found with flexion of the lumbar spine. In contrast, the FP subgroup achieved a comparable range of flexion to the no-LBP subgroup while also displaying an absence of FRP (Table 7). Ironically, the FP patients reported that flexion aggravated their LBP while extension relieved it. It is possible that these patterns of increased muscular activity of the sLM may have resulted from reflex muscle activation caused by stressing pain sensitive structures. ${ }^{51}$

Function 2 maximally separated the 2 subgroups of NS-CLBP and was dominated by the variables quantifying the subject's posture during usual sitting (lumbar angle). Univariate analysis showed that the AEP and FP subgroups tended to sit at opposing ends of the lumbar posture spectrum (hyperlordotic and kyphotic, respectively). This was in contrast with the no-LBP subjects who adopted a more neutral lumbar spine posture ${ }^{18,19}$ (Table 7). Previous studies have also demonstrated similar habitual loading into end range spinal flexion in the FP subgroup in both cyclists ${ }^{20}$ and industrial workers with CLBP. ${ }^{21}$ Scannell and McGill ${ }^{52}$ proposed that individuals with a hypolordotic and those with a hyperlordotic lumbar posture load different regions of the passive tissue angle-torque deformation curve. Loading near the end of the curve in turn increases the potential for tissue strain and pain.

The 2 clinical subgroups presented with a consistent pattern of altered motor control in both static (sitting) and dynamic (forward bending) conditions. These results when linked to their clinical data support the concept that these patients present with a maladaptive motor control pattern with the potential to act as an underlying mechanism for ongoing strain and potential peripheral nociceptive generation (pain). ${ }^{43}$ These findings are consistent with Scannell and $\mathrm{McGill}^{53}$ who reported that having a history of low back disorders was found to be associated with workers moving and activating their muscles in a manner that resulted in higher spinal loads. The authors also suggested that this pattern would increase their risk of sustaining tissue damage, potentially hindering their recovery. ${ }^{53,54}$

In this study, the pain intensity, disability, and fearavoidance beliefs were similar in both NS-CLBP subgroups, suggesting that these factors were not related to driving the different motor control patterns observed in the 2 pain subgroups.

\section{Clinical Implications}

This study demonstrated that a series of tests, involving analysis of posture (static) and movements (dynamic) reported as aggravating LBP in the patients, discriminated between the 2 patient subgroups and the pain-free controls, previously clinically identified by 2 independent clinicians. This finding supports the validity of clinical process of diagnostics, where a diagnosis is determined as a result of a complete physical evaluation involving a series of tests while closely correlating these findings to the individual's complaints. ${ }^{15,23,43}$

The validity of clinical examination to diagnose/ classify LBP has previously been questioned. Bogduk and McGuirk $^{55}$ stated there was a lack of evidence to believe that any particular clinical sign, or combination of signs, found by the process of examination allows a valid or reliable diagnosis. Although it is acknowledged that the "signs and symptoms" of altered motor control without the process of diagnostics are potentially meaningless, the results of this study suggest that analysis of postures and movements that form the basis to subclassify (when correlated to the patients pain behavior), are "real phenomena" in NS-CLBP patients with motor control impairment.

The results highlight the heterogeneity of the NSCLBP population with regards to motor control of the lumbar spine and the potential importance of classification in the identification of subgroups. These findings also have potentially important implications for therapeutic management. A motor learning intervention ${ }^{15} \mathrm{di}^{-}$ rected at changing maladaptive movement behavior to unload sensitized spinal structures, seems a logical approach when considering these data. This approach represents a cognitive movement-based intervention, aimed at teaching pain control by altering maladaptive movement behavior while enhancing functional capacity. ${ }^{15}$ Previous research into this motor learning approach to managing specific subgroups of CLBP patients has been shown to be effective. ${ }^{56}$

\section{Implications for Clinical Research}

This study provides support for the classification of NSCLBP patients with motor control impairment. The heterogeneity of the NS-CLBP population provides further support for the need to identify and use subgroups in clinical research. For example, recent studies examining sitting in NS-CLBP patients have demonstrated a "washout effect" where the findings in one subgroup of patients were "washed-out" by the opposite findings of patients belonging to another subgroup when classification was not applied. ${ }^{18,19}$

To gain a better understanding of the different and complex aspects of altered motor control in NS-CLBP, the findings of this study support the approach taken, where both clinical and laboratory (trunk muscle activation and lumbosacral kinematics) data are combined. 
Spinal kinematic and sEMG data are deemed critical to provide an insight into the functional spinal postures adopted in the different subgroups relative to the spine's neutral and elastic zones. ${ }^{57,58}$ This in turn provides speculative insight into the relative loading of the passive spinal structures.

The development of a series of tests that discriminate patients with motor control impairment provides the basis for further investigations into treatment outcomes and the role of motor control impairment in the generation and maintenance of LBP. Such investigations have the potential to provide greater insight into the cause/ effect dilemma of altered motor control and its relationship to LBP. ${ }^{59}$

An advantage of using a classification method incorporating a series of functional tests for clinical research into NS-CLBP is that these tasks are not as susceptible to the inaccuracies observed by volitional control. ${ }^{60,61}$ This is supported by the excellent reliability that was observed between testing sessions. Although simple functional measures such as gross trunk ROM, the coordination of different regions of the spine and pelvis is a characteristic that is largely independent of willful regulation. ${ }^{60,61}$ As only low-level exertions (e.g., submax MVIC) are required for this type of testing, this makes it widely applicable in LBP populations.

\section{Limitations}

A potential limitation of this study was the use of clinicians as the gold standard. However, as the aim of the study was to assess the validity of this clinical decisionmaking process, it was necessary and reflects the reality of clinical practice. In this context it is acknowledged that good performance of the Statistical Classification Model refers to good prediction of the gold standard clinical classification. ${ }^{61}$

Further, the information regarding the level of muscle activation is limited to the superficial muscles assessed via surface EMG. Intramuscular EMG techniques are required to determine changes in the deep spinal stabilizing muscles and to gain a more complete understanding of the relevance of all trunk muscles (and their dysfunction) in LBP populations.

It is further acknowledged that the application of strict inclusion/exclusion criteria limits the generalisability of our results to patients with an AEP and FP classification. Further studies should include other patterns of motor control impairment as described by O'Sullivan. ${ }^{15}$ Other patterns were not tested for logistical reasons and convenience of sampling. It is possible that the Statistical Classification Model could be customized (using different parameters) to other motor control impairment patterns and to other LBP subgroups.

The authors acknowledge that further validation studies are required for the overall clinical validation of the classification system. It is essential to test the efficacy of incorporating the classification model in the decisions of selecting specific treatment based on the underlying mechanism. It will also be important to test the validity by means of outcome studies (RCT); such validation is currently underway.

It was beyond the scope of this study to reveal the answer to the "chicken or egg" dilemma regarding "altered motor control and LBP." From this perspective it is essential to do more longitudinal studies (e.g., looking at patients for a longer period of time during the day) or prospective studies and investigate if LBP causes altered motor control or altered motor control generates LBP.

\section{- Conclusion}

A Statistical Classification Model using laboratorybased methods to quantify parameters of motor control demonstrated that the differences between no-LBP, AEP, and FP subgroups are real phenomena and support the clinical decision-making process of trained clinicians. The altered motor control patterns identified, when linked to the clinical data, represent potentially provocative and maladaptive movement behavior that represents a potential mechanism for peripheral nociceptive drive of pain. These results increase the evidence for the validity of the proposed classification system.

\section{- Key Points}

- A Statistical Classification Model demonstrated that 2 clinical subgroups of NS-CLBP patients with MCI can be discriminated from asymptomatic controls, and each other, using selected parameters of motor control.

- The data support the concept of NS-CLBP patients presenting with maladaptive motor control. - The differences in trunk muscle activation and lumbosacral kinematics in 2 subgroups of NSCLBP found in this study strengthens the hypothesis that there exist distinctly different underlying mechanisms of pain.

- The results highlight the heterogeneity of motor control of the NS-CLBP population and this may have important implications for clinical research therapeutic management.

\section{References}

1. Ehrlich GE. Low back pain. Bull World Health Organ 2003;81:671-6.

2. Woolf AD, Pfleger B. Burden of major musculoskeletal conditions. Bull World Health Organ 2003;81:646-56.

3. Croft PR, Macfarlane GJ, Papageorgiou AC, et al. Outcome of low back pain in general practice: a prospective study. BMJ 1998;316:1356-9.

4. Burton AK, McClune TD, Clarke RD, et al. Long-term follow-up of patients with low back pain attending for manipulative care: outcomes and predictors. Man Ther 2004;9:30-5.

5. Dillingham T. Evaluation and management of low back pain: an overview. State Art Rev 1995;9:559-74.

6. Waddell G. The Back Pain Revolution. Edinburgh, United Kingdom: Churchill Livingstone; 2004.

7. Leboeuf-Yde C, Hennius B, Rudberg E, et al. Chiropractic in Sweden: a short description of patients and treatment. J Manipulative Physiol Ther 1997;20: 507-10. 
8. Leboeuf-Yde C, Manniche C. Low back pain: time to get off the treadmill. J Manipulative Physiol Ther 2001;24:63-6.

9. Borkan JM, Koes B, Reis S, et al. A report from the second international forum for primary care research on low back pain: reexamining priorities. Spine 1998;23:1992-6.

10. Borkan J, Van Tulder M, Reis S, et al. Advances in the field of low back pain in primary care: a report from the fourth international forum. Spine 2002; 27:E128-32.

11. Ford J. Classification of Low Back Pain (Faculty of Medicine, Dentistry and Health Science) [doctoral thesis]. Melbourne, Australia: The University of Melbourne; 2002.

12. Ford J, Story I, McKeenen J. A systematic review on methodology of classification system research for low back pain. In: Musculoskeletal Physiotherapy Australia 13th Biennial Conference; 2003; Sydney, Australia.

13. Petersen T. Non-Specific Low Back Pain-Classification and Treatment (Department of Physical Therapy) [doctoral thesis]. Lund, Sweden: Lund University; 2003.

14. McCarthy CJ, Arnall F, Strimpakos N, et al. The biopsychosocial classification of non-specific low back pain: a systematic review. Phys Ther Rev 2004;9:17-30.

15. O'Sullivan P. Clinical instability of the lumbar spine: its pathological basis, diagnosis, and conservative management. In: Boyling JA, Jull GA, eds. Grieve's Modern Manual Therapy. 3rd ed. Amsterdam, The Netherlands: Elsevier; 2004:311-31.

16. O'Sullivan PB. Lumbar segmental "instability": clinical presentation and specific stabilizing exercise management. Man Ther 2000;5:2-12.

17. Dankaerts W, O'Sullivan PB, Straker LM, et al. The inter-examiner reliability of a classification method for non-specific chronic low back pain patients with motor control impairment. Man Ther 2006;2:28-39.

18. Dankaerts W, O'Sullivan P, Burnett A, et al. Differences in sitting posture are associated with non-specific chronic low back pain disorders when patients are sub-classified. Spine 2006;31:698-704.

19. Dankaerts W, O'Sullivan P, Burnett A, et al. Altered patterns of superficial trunk muscle activation during sitting in non-specific chronic low back pain: importance of sub-classification. Spine 2006;31:2017-23.

20. Burnett AF, Cornelius MW, Dankaerts W, et al. Spinal kinematics and trunk muscle activity in cyclists: a comparison between healthy controls and nonspecific chronic low back pain subjects: a pilot investigation. Man Ther 2004;9:211-9.

21. O'Sullivan P, Mitchell T, Bulich P, et al. The relationship between posture, back muscle endurance, and low back pain in industrial workers. Man Ther 2006;11:264-71.

22. O'Sullivan PB, Burnett A, Floyd AN, et al. Lumbar repositioning deficit in a specific low back pain population. Spine 2003;28:1074-9.

23. Elvey R, O'Sullivan P. A contemporary approach to manual therapy. In: Boyling JA, Jull GA, eds. Grieve's Modern Manual Therapy. 3rd ed. Amsterdam, The Netherlands: Elsevier; 2004:471-94.

24. McGill SM, Cholewicki J, Peach JP. Methodological considerations for using inductive sensors (3SPACE ISOTRAK) to monitor 3-D orthopaedic joint motion. Clin Biomech (Bristol, Avon) 1997;12:190-4.

25. Callaghan JP, Gunning JL, McGill SM. The relationship between lumbar spine load and muscle activity during extensor exercises. Phys Ther 1998; 78:8-18.

26. Peach JP, Sutarno CG, McGill SM. Three-dimensional kinematics and trunk muscle myoelectric activity in the young lumbar spine: a database. Arch Phys Med Rehabil 1998;79:663-9.

27. Callaghan JP, McGill SM. Low back joint loading and kinematics during standing and unsupported sitting. Ergonomics 2001;44:280-94.

28. Green JP, Grenier SG, McGill SM. Low-back stiffness is altered with warm-up and bench rest: implications for athletes. Med Sci Sports Exerc 2002;34:1076-81.

29. Hudson-Cook N, Tomes-Nicholson K, Breen A. A revised Oswestry Disability Questionnaire. Manchester, United Kingdom: Manchester University Press; 1989.

30. Kori SH, Miller RP, Todd DD. Kinesiophobia: a new view of chronic pain behavior. Pain Manage 1990;3:35-43.

31. Pearcy M, Hindle RJ. New method for the non-invasive three-dimensional measurement of human back movement. Clin Biomech (Bristol, Avon) 1989; 4:73-9.

32. Dolan P, Greenfield K, Nelson RJ, et al. Can exercise therapy improve the outcome of microdiscectomy? Spine 2000;25:1523-32.

33. Hermens HJ, Freriks B, Disselhorst-Klug C, et al. Development of recommendations for SEMG sensors and sensor placement procedures. J Electromyogr Kinesiol 2000;10:361-74.
34. Abbott J. The nexus of clinical examination and outcome research. In: 8th International Federation of Orthopaedic Manipulative Therapists' Conference; 2004; Cape Town, South Africa.

35. Ng JK, Kippers V, Richardson CA. Muscle fibre orientation of abdominal muscles and suggested surface EMG electrode positions. Electromyogr Clin Neurophysiol 1998;38:51-8.

36. De Foa JL, Forrest W, Biedermann HJ. Muscle fibre direction of longissimus, iliocostalis, and multifidus: landmark-derived reference lines. J Anat 1989; 163:243-7.

37. Danneels LA, Cagnie BJ, Cools AM, et al. Intra-operator and inter-operator reliability of surface electromyography in the clinical evaluation of back muscles. Man Ther 2001;6:145-53.

38. Winter DA. Electromyogram recording, processing, and normalization: procedures and considerations. J Hum Muscle Perform 1991;1:5-15.

39. Dankaerts W, O'Sullivan PB, Burnett AF, et al. Reliability of EMG measurements for trunk muscles during maximal and sub-maximal voluntary isometric contractions in healthy controls and CLBP patients. J Electromyogr Kinesiol 2004;14:333-42.

40. Portney LG, Watkins MP. Foundations of Clinical Research: Applications to Practice. 2nd ed. Upper Saddle River, NJ: Prentice Hall; 2000.

41. Johnson R, Wichern D. Applied Multivariate Statistical Analysis. 5th ed. Upper Saddle River, NJ: Prentice Hall; 2002.

42. Otto M. Chemometricsed. Weinheim, Germany: Wiley-VCH; 1999.

43. O'Sullivan P. Diagnosis and classification of chronic low back pain disorders: maladaptive movement and motor control impairments as underlying mechanism. Man Ther. In press.

44. McKenzie R. The Lumbar Spine: Mechanical Diagnosis and Therapy. Waikanae, New Zealand: Spinal Publications Limited; 1989.

45. Delitto A, Erhard RE, Bowling RW. A treatment-based classification approach to low back syndrome: identifying and staging patients for conservative treatment. Phys Ther 1995;75:470-85; discussion 85-9.

46. Sahrmann SA. Diagnosis and Treatment of Movement Impairment Syndromes. St Louis, France: Mosby; 2001.

47. Riddle DL. Classification and low back pain: a review of the literature and critical analysis of selected systems. Phys Ther 1998;78:708-37.

48. Watson PJ, Booker CK, Main CJ, et al. Surface electromyography in the identification of chronic low back pain patients: the development of the flexion relaxation ratio. Clin Biomech (Bristol, Avon) 1997;12:165-71.

49. Kaigle AM, Wessberg P, Hansson TH. Muscular and kinematic behavior of the lumbar spine during flexion-extension. J Spinal Disord 1998;11:163-74.

50. Kavcic N, Grenier S, McGill SM. Determining the stabilizing role of individual torso muscles during rehabilitation exercises. Spine 2004;29:1254-65.

51. Solomonow M, Baratta RV, Zhou BH, et al. Muscular dysfunction elicited by creep of lumbar viscoelastic tissue. J Electromyogr Kinesiol 2003;13: 381-96.

52. Scannell JP, McGill SM. Lumbar posture-should it, and can it, be modified? A study of passive tissue stiffness and lumbar position during activities of daily living. Phys Ther 2003;83:907-17.

53. McGill S, Grenier S, Bluhm M, et al. Previous history of LBP with work loss is related to lingering deficits in biomechanical, physiological, personal, psychosocial, and motor control characteristics. Ergonomics 2003;46:731-46.

54. McGill SM. Linking latest knowledge of injury mechanisms and spine function to the prevention of low back disorders. J Electromyogr Kinesiol 2004; $14: 43-7$.

55. Bogduk N, McGuirk B. Medical Management of Acute and Chronic Low Back Pain: An Evidence Based Approach. Pain Research and Clinical management. Amsterdam, The Netherlands: Elsevier; 2002;41-7.

56. O'Sullivan PB, Twomey LT, Allison GT. Evaluation of specific stabilizing exercise in the treatment of chronic low back pain with radiologic diagnosis of spondylolysis or spondylolisthesis. Spine 1997;22:2959-67.

57. Panjabi M, Abumi K, Duranceau J, et al. Spinal stability and intersegmental muscle forces: a biomechanical model. Spine 1989;14:194-200.

58. Panjabi MM. The stabilizing system of the spine, part II. Neutral zone and instability hypothesis. J Spinal Disord 1992;5:390-6.

59. Cholewicki J, van Dieen JH, Arsenault AB. Muscle function and dysfunction in the spine. J Electromyogr Kinesiol 2003;13:303-14.

60. Leclaire R, Esdaile JM, Jequier JC, et al. Diagnostic accuracy of technologies used in low back pain assessment: thermography, triaxial dynamometry, spinoscopy, and clinical examination. Spine 1996;21:1325-30; discussion 31.

61. Marriott A, Newman NM, Gracovetsky SA, et al. Improving the evaluation of benign low back pain. Spine 1999;24:952-60. 